\title{
The role of wax and resin in the nestmate recognition system of a stingless bee, Tetragonisca angustula
}

\author{
Sam M. Jones • Jelle S. van Zweden • Christoph Grüter • Cristiano Menezes • \\ Denise A. Alves • Patrícia Nunes-Silva • Tomer Czaczkes • \\ Vera L. Imperatriz-Fonseca • Francis L. W. Ratnieks
}

Received: 27 May 2011 /Revised: 4 August 2011 /Accepted: 18 August 2011 /Published online: 20 September 2011

(C) Springer-Verlag 2011

\begin{abstract}
Recent research has shown that entrance guards of the stingless bee Tetragonisca angustula make less errors in distinguishing nestmates from non-nestmates than all other bee species studied to date, but how they achieve this is unknown. We performed four experiments to investigate nestmate recognition by entrance guards in T. angustula. We first investigated the effect of colony odours on acceptance. Nestmates that acquired odour from nonnestmate workers were $63 \%$ more likely to be rejected while the acceptance rate of non-nestmates treated with nestmate odour increased by only $7 \%$. We further hypothesised that guards standing on the wax entrance tube might use the tube as an odour referent. However, our findings showed that there was no difference in the acceptance of non-nestmates by guards standing on their own colony's
\end{abstract}

Communicated by W. Hughes

Electronic supplementary material The online version of this article (doi:10.1007/s00265-011-1246-7) contains supplementary material, which is available to authorized users.

S. M. Jones $(\bowtie) \cdot$ J. S. van Zweden • C. Grüter • T. Czaczkes •

F. L. W. Ratnieks

Laboratory of Apiculture and Social Insects,

School of Life Sciences, University of Sussex,

Falmer,

Brighton BN1 9QG, UK

e-mail: sj203@sussex.ac.uk

C. Menezes $\cdot$ D. A. Alves $\cdot$ P. Nunes-Silva .

V. L. Imperatriz-Fonseca

Faculdade de Filosofia, Ciências e Letras de Ribeirão Preto, USP, Avenida Bandeirantes nr, 3900, Monte Alegre,

Ribeirão Preto, SP, Brazil

D. A. Alves

Departamento de Ecologia, Instituto de Biociências, USP,

Rua do Matão, trav. 14 nr. 321, Cidade Universitária,

São Paulo, Brazil entrance tube versus the non-nestmate's entrance tube. Moreover, treatment of bees with nestmate and nonnestmate resin or wax had a negative effect on acceptance rates of up to $65 \%$, regardless of the origin of the wax or resin. The role of resin as a source of recognition cues was further investigated by unidirectionally transferring resin stores between colonies. Acceptance rates of nestmates declined by $37 \%$ for hives that donated resin, contrasting with resin donor hives where acceptance of non-nestmates increased by $21 \%$. Overall, our results confirm the accuracy of nestmate recognition in $T$. angustula and reject the hypothesis that this high level of accuracy is due to the use of the wax entrance tubes as a referent for colony odour. Our findings also suggest that odours directly acquired from resin serve no primary function as nestmate recognition cues.

Present Address:

J. S. van Zweden

Centre for Social Evolution, University of Copenhagen,

Universitetsparken 15,

2100 Copenhagen, Denmark

Present Address:

C. Menezes

Embrapa Amazônia Oriental,

Trav. Dr. Enéas Pinheiro s/ nr.,

Belém, Para, Brazil

Present Address:

V. L. Imperatriz-Fonseca

Universidade Federal Rural do Semiárido,

Mossoró, Rio Grande do Norte, Brazil 
The lack of consistency among colonies plus the complex results of the third and fourth experiments highlight the need for further research on the role of nest materials and cuticular profiles in understanding nestmate recognition in T. angustula.

Keywords Jataí $\cdot$ Meliponini $\cdot$ Stingless bees $\cdot$ Recognition template $\cdot$ Nestmate recognition

\section{Introduction}

Recognition of self versus non-self is ubiquitous among organisms, operating at several different levels and involving a variety of mechanisms (e.g. Beale 1990; Janeway and Medzhitov 2002; Nasrallah 2002; Glass and Kaneko 2003). Eusocial insects demonstrate self versus non-self recognition predominantly at the colony level (but see Tibbetts 2002; d'Ettorre and Heinze 2005). In many species, the nest entrance is defended by guards who deter both allospecific and conspecific intruders (Butler and Free 1952; Bell et al. 1974; Wittmann et al. 1990). Conspecific recognition requires the matching of a set of cues carried on the cuticle of an encountered individual (the label) with a previously acquired representation of colony odour (the template) of an evaluating individual (van Zweden and d'Ettorre 2010). Depending on the degree of similarity/dissimilarity, the encountered conspecific is accepted or rejected (Lacy and Sherman 1983; Vander Meer et al. 1998). Ideally, nestmate recognition should categorise all incoming individuals without error (Sherman et al. 1997), but mistakes are made: nestmates may be rejected (rejection errors) or non-nestmates admitted (acceptance errors). Which of these two errors is minimised can vary adaptively via adjustment of the acceptance threshold. For example, increased rejection errors may be traded off for decreased acceptance errors when the frequency of intruders or the cost of admitting them is higher (Reeve 1989; Downs and Ratnieks 2000; Couvillon et al. 2009).

In the honeybee, Apis mellifera, the number of entrance guards and the permissiveness of the acceptance threshold change adaptively, depending on nectar availability and robbing intensity (Downs and Ratnieks 2000; Couvillon et al. 2008). Overall, the recognition error rates are surprisingly high, with means of approximately $23 \%$ (range 8 $48 \%$ ) for rejection errors and $29 \%$ (range $21-62 \%$ ) for conspecific acceptance errors (Breed 1983; Downs and Ratnieks 1999, 2000; Couvillon et al. 2007a, 2008, 2009, 2010). This gives a total error of approximately $52 \%$, almost exactly midway between the two extremes of perfect $(0 \%)$ and zero information (100\%) (Ratnieks 1991). This is in stark contrast to recent results for the Neotropical stingless bee, Tetragonisca angustula. Guards of T. angus- tula made few errors in discriminating nestmate workers from non-nestmate conspecifics, accepting all nestmate workers ( $0 \%$ rejection errors) while rejecting $92 \%$ of conspecific non-nestmate workers, giving a total error of only 8\% (Kärcher and Ratnieks 2009). This is also considerably lower than the error rates reported for five other Neotropical stingless bees (Table 1).

This raises the question on what the underlying mechanisms are that allow T. angustula to have lower recognition error rates than honeybees or other stingless bees. One obvious difference between $T$. angustula and the six other bee species is that $T$. angustula is the only one that constructs a wax entrance tubes for its nest. Nests of Frieseomelitta varia have a round entrance hole surrounded by resin, nests of Trigona fulviventris have wide, sometimes tubular, resin openings, while those of the three Melipona species, Melipona quadrifasciata, Melipona rufiventris and Melipona scutellaris, all possess a small entrance hole surrounded by dry mud (Roubik 2006; Couvillon et al. 2007b; M.J. Couvillon, personal communication; S.M. Jones, personal observation). Wax is important in honey bee recognition, functioning as the primary source of colony odour cues and a wax entrance tube might provide guards with a more direct template with which to compare incoming bees (Breed et al. 2004; Couvillon et al. 2007a). This might allow guards to update their template more frequently to allow peripheral sensory detection via desensitisation (c.f. Ozaki et al. 2005) or to simply enable a direct comparison.

A further difference between $T$. angustula and $A$. mellifera is the former's greater use of plant resins. Leonhardt et al. (2009) recently demonstrated that terpenoid profiles, derived from resin, extracted from the cuticles of seven Paleotropical stingless bee species varied quantitatively between colonies of the same species, leading them to suggest that this may potentially serve some communicative function in these stingless bee species. This is entirely feasible given that conspecific recognition may rely on quantitative differences within the same set of compounds (vander Meer et al. 1989; Espelie et al. 1990; Martin et al. 2008; van Zweden and d'Ettorre 2010). Nests of $T$. angustula contain substantial amounts of resin stored in numerous piles throughout the nest. Under a microscope (magnification $\times 240$ ), resin can also be seen in a layer on the legs, head and thorax of foragers (J.S. van Zweden, unpublished data) and is also mixed with wax to form cerumen, which is used to construct the combs and surrounding involucrum (Nogueira-Neto 1997; S.M. Jones, personal observation; Wille 1983). Thus, the ubiquitous presence of resin within the nest, either in its pure form as piles or as cerumen, should be sufficient for acquisition of a colony-encompassing odour profile. Indeed this would in many ways be analogous to the ubiquitous presence of wax in the combs of honeybees, although wax is secreted by the 
Table 1 Error rates for T. angustula and six other bee species

\begin{tabular}{lllll}
\hline Bee species & $\begin{array}{l}\text { Rejection } \\
\text { error rate (\%) }\end{array}$ & $\begin{array}{l}\text { Acceptance } \\
\text { error rate (\%) }\end{array}$ & $\begin{array}{l}\text { Total error } \\
\text { rate (\%) }\end{array}$ & Reference(s) \\
\hline Apis mellifera & 33 & 31 & 64 & Breed 1983 \\
& 26 & $18-30$ & $44-56$ & Couvillon et al. 2007a \\
& $26-48$ & $30-59$ & $56-107$ & Couvillon et al. 2008 \\
& $19-24$ & $57-62$ & $76-86$ & Couvillon et al. 2009 \\
& 8 & 30 & 38 & Couvillon et al. 2010 \\
Frieseomelitta varia & 18 & 21 & 39 & Downs and Ratnieks 1999 \\
Melipona quadrifasciata & 0 & 22 & 39 & Downs and Ratnieks 2000 \\
Melipona rufiventris & 0 & 27 & 38 & Couvillon and Ratnieks 2008 \\
Melipona scutellaris & 0 & 26 & 26 & Breed and Page 1991 \\
Tetragonisca angustula & 0 & 86 & 86 & Breed and Page 1991 \\
Trigona fulviventris & 24 & 40 & 40 & Breed and Page 1991 \\
\hline
\end{tabular}

bees while resin is collected (Breed et al. 1995; d'Ettorre et al. 2006).

The aim of this study was to investigate conspecific recognition in $T$. angustula, with emphasis on the effects of odours derived from wax entrance tubes, plant resins and worker bees. This was achieved by investigating whether the acceptance of introduced nestmates and non-nestmates by guards standing on the entrance tube was influenced by: (1) the acquisition of cuticular odours derived from nestmates and non-nestmates onto the cuticle, (2) swapping wax entrance tubes between colonies, (3) the acquisition of resin and wax derived from nestmates and non-nestmates onto the cuticle and (4) the unidirectional swap of entire resin stores between hives.

\section{Methods}

Study site and organism

Data were collected in February 2009 (experiments 1 and 2) and 2010 (experiments 3 and 4) at Fazenda Aretuzina, São Simão, São Paulo State, Brazil. During both study periods, the weather was hot, with daytime high temperatures of ca. $24-32^{\circ} \mathrm{C}$ and periodic heavy rain. Data were only collected on non-rainy days during active foraging (between 9.00 and 17.00 hours).

T. angustula, local name Jataí, is unique among the stingless bees in possessing two types of guards: both hovering and standing (van Zweden et al. 2011). Hovering guards flank the flight path leading to the nest and readily attack allospecific bees approaching the nest vicinity (Wittmann 1985; Grüter et al. 2011), thus increasing the defensive perimeter of the nest (van Zweden et al. 2011). While hovering guards are efficient at detecting individuals visually dissimilar to themselves, it is the role of the standing guards, which stand around the opening on the tip of the entrance tube, to distinguish non-nestmate conspecifics from nestmates, which they do by contact chemoreception (Kärcher and Ratnieks 2009). The two types of guards complement one another and increase the defensive efficiency of the colony.

We studied five colonies of $T$. angustula in 2009 (experiments 1 and 2) and six in 2010 (experiments 3 and 4). Each colony was housed in a wooden hive box (ca. 50 -cm high $\times$ $20 \mathrm{~cm} \times 30 \mathrm{~cm}$ ), with a circular entrance hole of $1.8 \mathrm{~cm}$ in diameter (for more details, see Nogueira-Neto (1997)). Each colony had built a wax entrance tube from this hole. Entrance tubes were ca. 1-3 cm long and had a circular opening at the tip ca. $0.6 \mathrm{~cm}$ in diameter (see also figures in Wittmann (1985); Couvillon et al. 2007b; for more detail, see Grüter et al. (2011)). The entrance tubes on the study colonies appeared identical to those of unmanaged colonies nesting in walls. Hives were raised ca. $1 \mathrm{~m}$ aboveground on hive stands or attached to the walls of buildings. The study colonies were queenright and thriving, with the hive nearly full of combs, covered by involucrum and numerous honey pots with a population of many thousands of workers. Mature colonies of $T$. angustula in Costa Rica were estimated to have approximately 10,000 workers (van Veen and Sommeijer 2000).

Introduction of worker bees to guards and their acceptance or rejection

The acceptance or rejection of conspecific workers by guards standing on the entrance tube was determined using a standard bioassay (Downs and Ratnieks 2000) developed for studying honey bees, $A$. mellifera, and modified for use with T. angustula (Kärcher and Ratnieks 2009). Returning 
foragers were collected at the hive entrance, placed in a tube and immediately chilled in an ice chest for 10-20 min, then removed one at a time and allowed to warm to ambient temperature. Once warmed, these workers walked actively but were less likely to fly than previously unchilled workers. A worker was taken from the ice chest and, once warmed up, allowed to grasp a clean wooden toothpick and walk onto the outer surface of the tip of the entrance tube of a discriminator hive. On contact with the guards standing on the entrance tube behaviour was observed for up to $2 \mathrm{~min}$. The introduced worker was considered "rejected" if it was bitten and tugged for the duration of the observation period or fell off the tube while grappling with a guard (Kärcher and Ratnieks 2009). The worker was considered "accepted"" if she was subjected only to licking and antennal contact or bitten and tugged for a few seconds and then left alone. Each time four bees were introduced in a row to a particular discriminator hive pseudorandomly with approximately $5 \mathrm{~min}$ between each introduction. Once the four bees had been introduced, the same protocol was repeated at the next discriminator hive, ensuring that a minimum of $45 \mathrm{~min}$ had passed before returning to a particular discriminator hive. The observer was unaware of the treatment group of the introduced workers. The number of standing guards present on the entrance tube was recorded before introductions commenced (mean \pm $\mathrm{SD}=15.43 \pm 4.38)$.

Experiment 1: the effect of bee-derived odours on acceptance rates of worker bees

The aim of this experiment was to determine how the transfer of nestmate and non-nestmate odours onto worker bees affected the acceptance of both nestmates and non-nestmates.

Four hives (A-D) were used both as discriminator and donor colonies. These were grouped into two pairs (A and $\mathrm{B}, \mathrm{C}$ and $\mathrm{D})$ to serve as donors and discriminators to each other (Fig. 1a). A fifth hive (E) was used as an additional source of non-nestmates. Worker bees $(n=20 \pm 3)$ were collected at hive entrances and placed in a $6-\mathrm{ml}$ clear plastic vial for $60 \mathrm{~min}$ to transfer odours to the vial at two vials per hive per study day. The bees were then released. Odours deposited on the inside of the tubes by these bees were then indirectly transferred to returning foragers by placing 12 individuals into a prepared vial for $15 \mathrm{~min}$. Each vial was used only once. Fresh vials were prepared on each study day and used within $4 \mathrm{~h}$.

The acceptance rate of the following seven treatments of workers were compared (Fig. 1a): (1) nestmates, untreated; (2) nestmates, treated with nestmate odour using the vial; (3) nestmates, treated with non-nestmate odour from the paired hive using the vial; (4) non-nestmates from the paired hive, untreated; (5) non-nestmates from hive E, untreated; (6) non-nestmates, treated with non-nestmate odour from the paired hive; and (7) non-nestmates, treated with nestmate odour.

Combined sample sizes for each of the seven treatments ranged between 99 and 111, with similar numbers introduced to each of the four discriminator hives.

Experiment 2: is the wax entrance tube used as a referent?

The aim of this experiment was to determine whether $T$. angustula guards use the wax entrance tube as a template or referent for colony odour. To achieve this, we swapped entrance tubes between paired colonies using the same pairings as in experiment 1 . The entrance tube was gently cut away from the hive entrance hole using a penknife. By using the natural stickiness of the wax, the entrance tube could be attached to the end of a 1.5 -cm-long plastic tube that exactly fitted into the hive entrance hole. The plastic tube was then placed into the hive's entrance hole and the colony was given 1-3 days for the entrance tube to attach firmly to the plastic tube using additional wax. Entrance tubes could then be swapped between hives in minutes, without physical damage and with minimal disturbance. Following tube swapping, guards appeared to behave normally on the new entrance tube.

The experimental design was the same as that used for experiment 1 , with the exception that acceptance rates were determined only for the following treatments: (1) nestmates, (2) paired hive non-nestmates and (3) hive E nonnestmates. These were compared before and 1,5 and $24 \mathrm{~h}$ after the swap (Fig. 1a).

Combined sample sizes ranged between 101 and 120 introduced bees for each of the three treatments both before and after the tube swap. Similar numbers were introduced to each of the four discriminator hives for each treatment.

Experiment 3: transfer of wax and resin odours to workers

The aim of this experiment was to determine if resin- or wax-derived recognition cues were utilised by $T$. angustula for the purpose of nestmate recognition. Six hives (F-K) were used: three discriminator colonies and three donor colonies for wax, resin and non-nestmates (Fig. 1b). Each discriminator colony thus received workers from all of the donor colonies in addition to their own nestmates. This change to the design from that used in experiments 1 and 2 diminishes any possible colony-specific effects as these can be dealt with statistically. Each discriminator hive received bees from its own hive and each of the three donor hives (i.e. a full factorial design) were treated as follows: (1) nestmates untreated, (2) nestmates treated with nestmate resin/wax, (3) nestmates treated with non-nestmate resin/wax, (4) non- 
Fig. 1 a Experimental design of experiments 1 and 2 . Treated and untreated worker bees were introduced to the entrance tubes of each discriminator hive.

There were two pairs of

discriminator hives (one black and one white). Hive E served as an additional source of non-nestmates, common to all four colonies. In the boxes on the arrow, unbracketed capital letters refer to the colony the introduced bee is from and the bracketed letters refer to the colony that the odour treatment, if any, originates from. $\mathbf{b}$ Experimental design of experiment 3. Treated and untreated worker bees were introduced to the three discriminator hives, $I, J$ and $K$, from the donor hives, $F$, $G$ and $H$. c Experimental design of experiment 4. Untreated worker bees were introduced to the entrance tubes of six discriminator hives $F-K$. Hives $F-H$ acted as "resin donors" and hives $I-K$ as "resin recipients". Resin was swapped unidirectionally from the donor hives to the recipient hives. Worker bees were subsequently introduced to all hives $24 \mathrm{~h}$ afterwards for a period of 7 days a

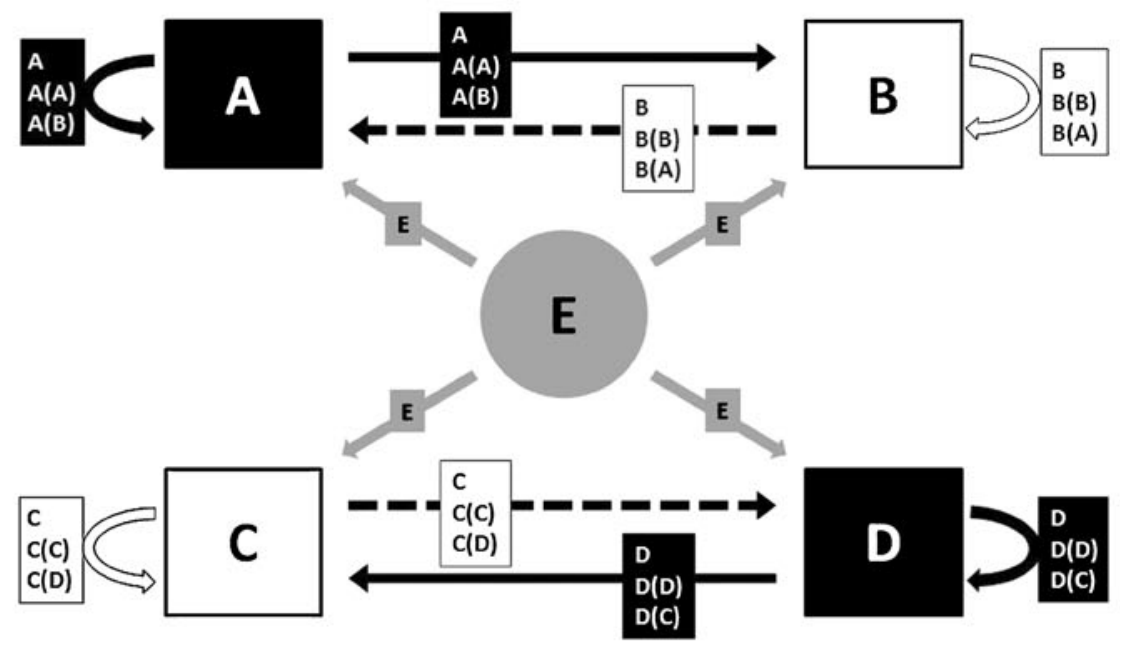

b

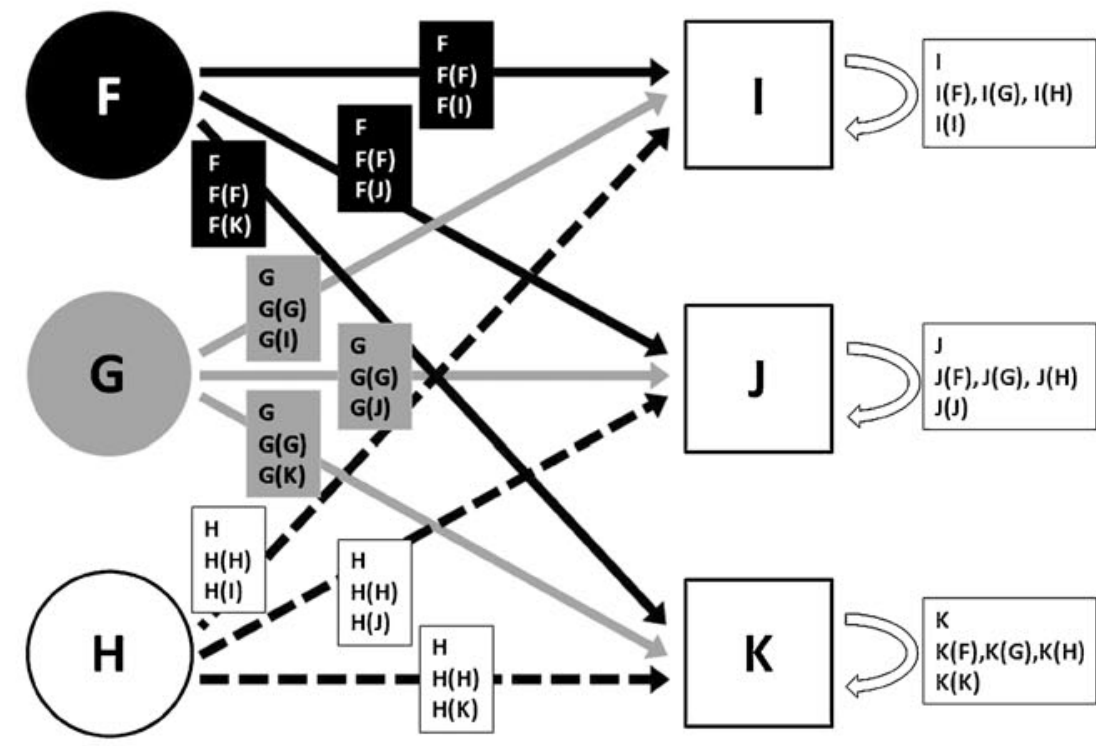

C

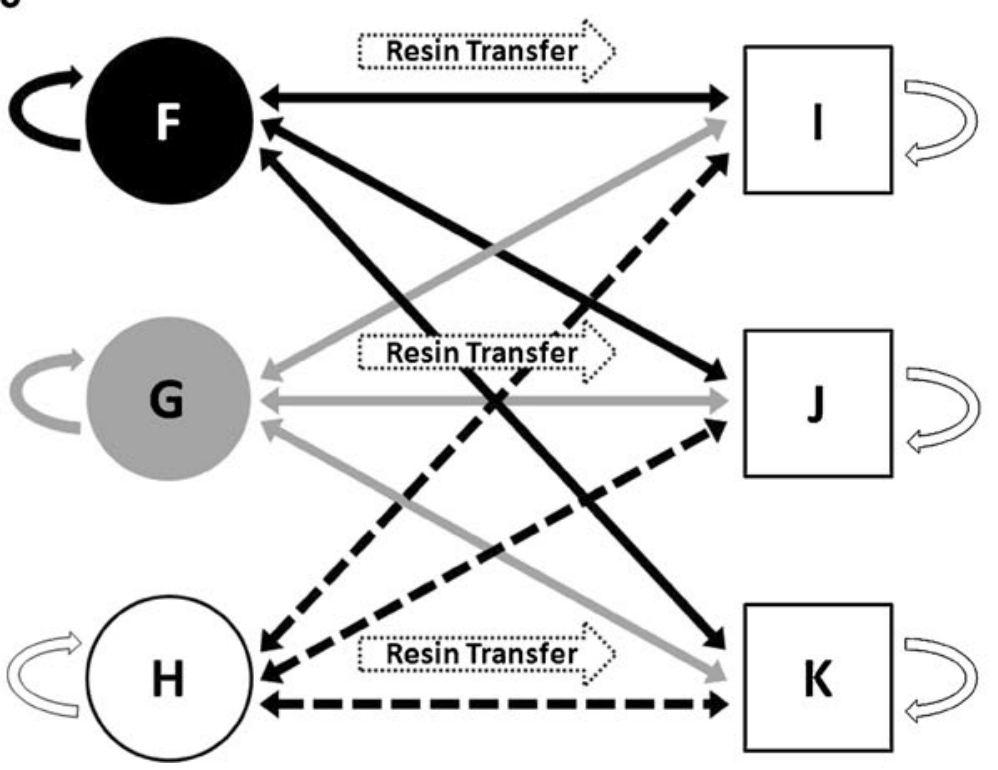


nestmates untreated, (5) Non-nestmates treated with nestmate resin/wax and (6) non-nestmates treated with non-nestmate resin/wax. Combined sample sizes per treatment ranged between 35 and 102 introduced bees with similar numbers introduced to the three discriminator hives.

Resin was collected from resin piles within each colony and white wax was collected from newly constructed entrance tubes. We are confident that the resin we collected from the piles contained little or no wax because its dark colour and viscous consistency was identical to the resin carried in the corbiculae of returning foragers. Moreover, Gastauer et al. (2011) observed no mixing of wax or other substances with the resin collected by worker bees of seven Neotropical stingless bee species, including $T$. angustula. Each 4-ml glass vial was treated with $0.5 \mathrm{ml}$ of hexane containing $2.5 \mathrm{mg}$ of either wax or resin. Evaporation left a thin, barely visible coating within each vial. Up to four workers were transferred to a treated vial and left for at least $15 \mathrm{~min}$ to allow indirect transfer. The bees were then chilled and introduced individually to the entrance tube of a discriminator colony as in experiment 1 . Each vial was used up to three times to treat a maximum of ten bees.

\section{Experiment 4: one-way transfer of resin between hives}

The aim of this experiment was to investigate the effect on the nestmate recognition label and/or template of unidirectional transfer of resin between $T$. angustula hives. Hive inspections showed that all the $T$. angustula nests had resin piles of varying sizes, all dark brown in colour, which were distributed throughout the nest. The mean weight of the entire resin reserves for the six colonies was $7.79 \pm 2.01 \mathrm{~g}$ (mean \pm 1 s.e., range $=2.04-16.05 \mathrm{~g}$ ).

Entire resin stores were removed from a donor hive, weighed and distributed as new piles within a receiving hive that had been cleared of existing resin piles the day before. Six colonies were used $(\mathrm{F}-\mathrm{K})$, paired up as three groups containing a 'resin donor' and 'resin acceptor' ( $F$ and I, G and J, H and K; Fig. 1c). Bees were introduced to all hives prior to and following the swap. Each donor hive received nestmates and non-nestmates from each of the three resin acceptor hives and vice versa for the receiving hives. Depending on the treatment, combined sample sizes ranged between 17 and 126 introduced bees. Introductions were undertaken at four different time periods: between 12 and $96 \mathrm{~h}$ before the resin transfer (control) and then at 12, 60 and $84 \mathrm{~h}$ after.

\section{Statistical analyses}

For data analysis, we used generalised linear mixed-effect models (GLMM) with binomial errors in R 2.9 (R Develop- ment Core Team 2009). We fitted the models using the lmer function (Bates 2007). Colony was included as a random effect throughout to control for the non-independence of data points from the same colony (Bolker et al. 2009; Zuur et al. 2009). For model selection, we used the protocol proposed by Zuur et al. (2009). We first explored the optimal structure of the random components by comparing random intercept models with random intercept and slope models. Then, we explored the optimal fixed component structure. Wald tests were used to determine the significance of the fixed effects (Bolker et al. 2009).

For all cases, the dependent variable was the response of the guards (accept or reject). The random variable was "discriminator colony" in all experiments. Fixed variables were "treatment" in experiment 1, "time (time following entrance tube swap)" in experiment 2, "treatment" and "origin" of bee (nestmate or non-nestmate) for experiment 3 and "treatment", "time" (before or after swap) and "origin" of bee for experiment 4 .

\section{Results}

Experiment 1: the effect of odour transfer on acceptance rates of worker bees

Guards standing on the entrance tubes made few recognition errors with untreated introduced bees, accepting significantly more nestmates than non-nestmates as expected (Fig. 2; $94.6 \%$ vs. $4.5 \%$, GLMM, Wald's $z=-14.15, p=<0.001)$. The strongest effect of odour treatment came from treating nestmates with non-nestmate odour, which resulted in an acceptance $54.3 \%$ lower than for nestmates treated with nestmate odour $(31.3 \%$ vs. $85.6 \%, z=-7.41, p=<0.001)$. Conversely, the acceptance rate of non-nestmates was not significantly affected by treatment; only $6.8 \%$ more nonnestmates were accepted when treated with nestmate odour than when treated with non-nestmate odour $(9.6 \%$ vs. $2.8 \%$, $z=1.92, p=0.054)$. A small but significant effect of the vial treatment itself could be seen on acceptance rates of bees treated with nestmate odour $(94.6 \%$ vs. $85.6 \%, z=-2.03$, $p=0.04$ ).

Experiment 2: is the wax entrance tube used as a referent?

Swapping entrance tubes did not affect the acceptance of either nestmates or non-nestmates (Fig. 2). There was no significant difference between the acceptance rates of nestmates for the four different time periods individually $(0 \mathrm{~h}$ vs. $1 \mathrm{~h}: z=-0.46, p=0.65 ; 0 \mathrm{~h}$ vs. $5 \mathrm{~h}: z=-1.83$, $p=0.067 ; 0 \mathrm{~h}$ vs. $24 \mathrm{~h}: z=0.14, p=0.91)$ and combined $(0 \mathrm{~h}$ vs. $1 / 5 / 24$ h: $94.6 \%$ vs. $90.8 \%, z=-1.05, p=0.29$ ). Similarly, there was no significant difference among the acceptance 

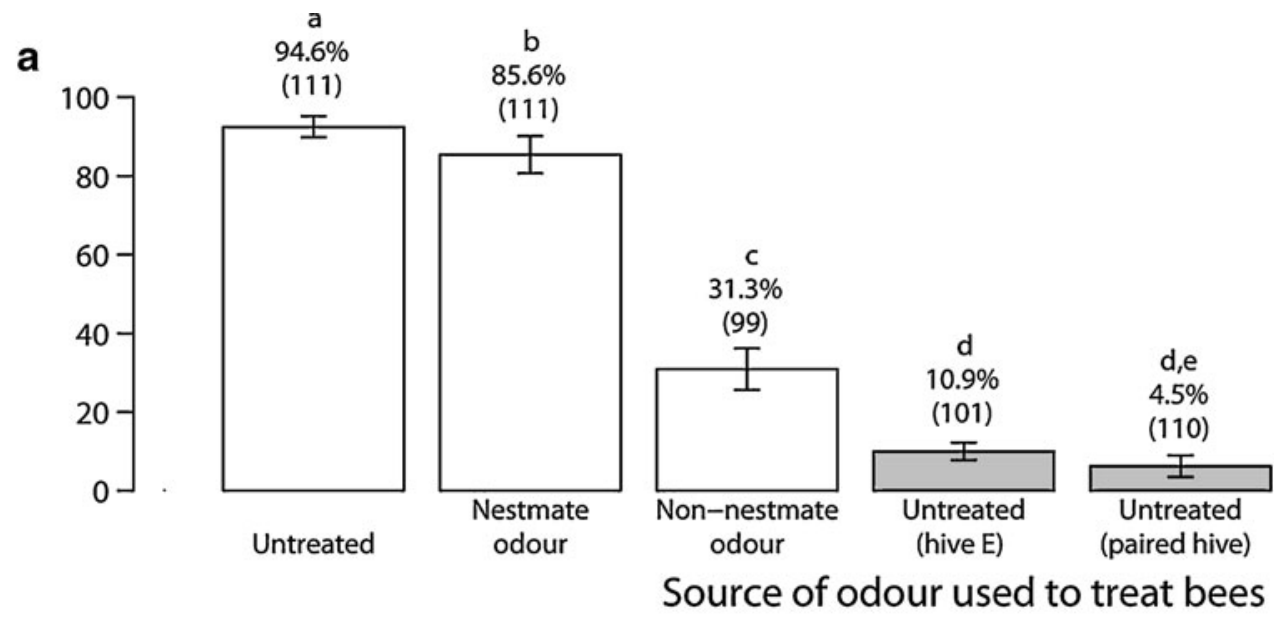

\section{Origin of bee \\ $\square$ Nestmate \\ $\square$ Non-nestmate}
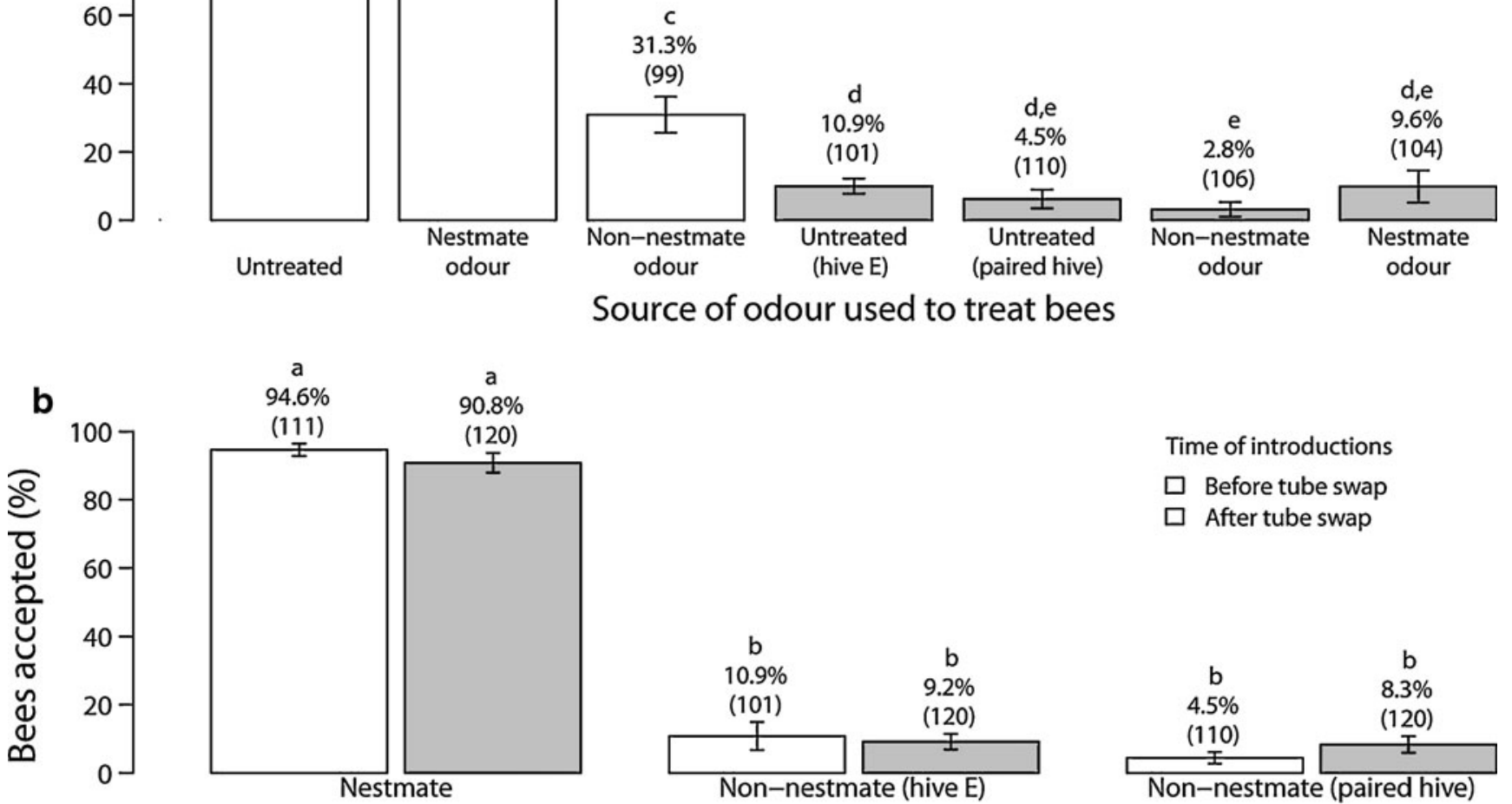

Origin of untreated bees introduced to discriminator hives
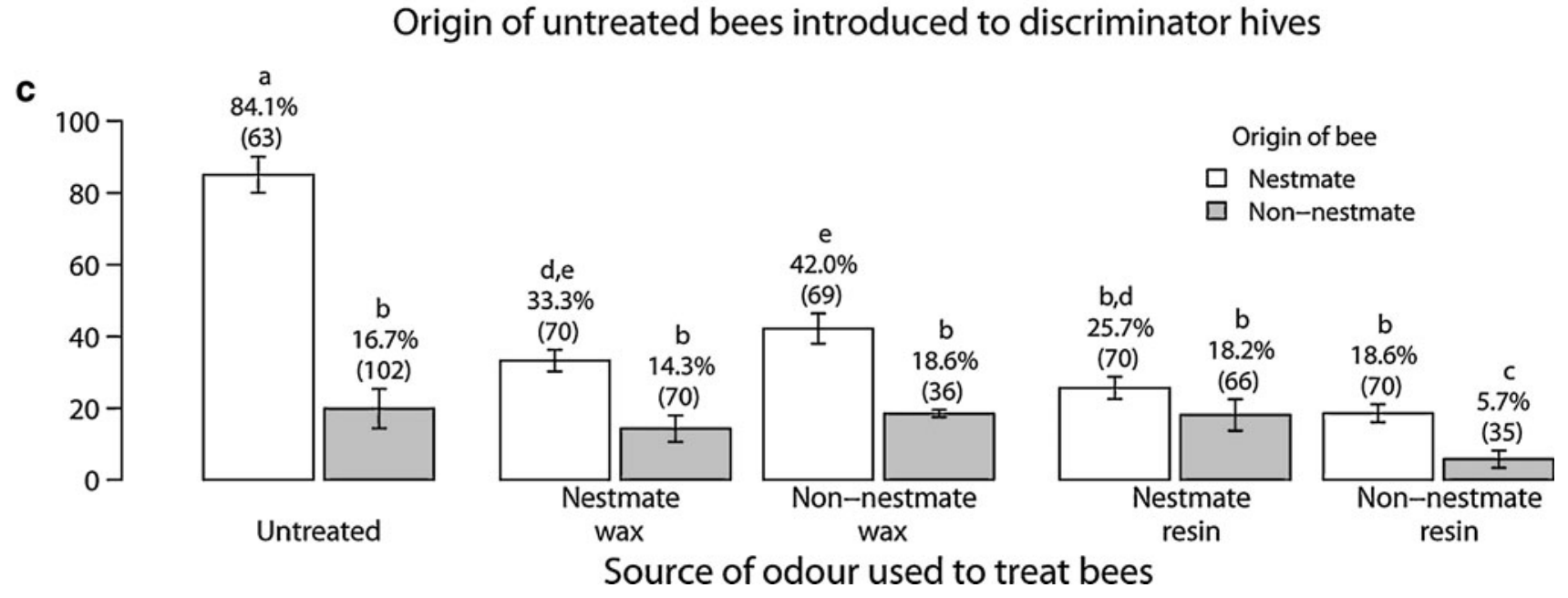

Time of introductions
$\square$ Before tube swap
$\square$ After tube swap

Fig. 2 Acceptance rates of introduced bees in experiments 1, 2 and 3. a Experiment 1: Treated and untreated worker bees were introduced to four discriminator hives, A, B, C and D. Non-nestmates introduced to discriminator hives originated from the paired hive and a fifth colony, colony E (untreated only), which served as a control. b Experiment 2: Nestmate workers and non-nestmate workers originating from both the paired hive and hive $\mathrm{E}$ (control) were introduced to four

rates of paired hive non-nestmates for the different time periods, both individually ( $0 \mathrm{~h}$ vs. $1 \mathrm{~h}: z=0.79, p=0.43 ; 0 \mathrm{~h}$ vs. 5 h: $z=0.79, p=0.43$; 0 h vs. $24 \mathrm{~h}: z=1.32, p=0.19$ ) and combined $(0 \mathrm{~h}$ vs. $1 / 5 / 24$ h: $4.5 \%$ vs. $8.3 \%, z=1.27$, discriminator hives, both before and after swapping the wax entrance tubes. c Experiment 3: Nestmates and non-nestmate workers, either untreated or treated, were introduced to three discriminator hives. Treated bees bore wax- or resin-derived odours from either their own hive or a foreign hive. Different letters denote significant differences. Exact percentage acceptance rates and sample sizes are given above the bars

$p=0.21$ ). As expected, there was also no change in the acceptance of non-nestmates from hive E post-swap $(0 \mathrm{~h}$ vs. $1 / 5 / 24$ h: $10.9 \%$ vs. $9.2 \%, z=1.26, p=0.21$ ). In addition, there is no indication that tube swapping affected the 
acceptance of non-nestmates from the paired colony any differently than non-nestmates from the control, hive E, with no significant interaction between treatment and tube swapping (pre-swap vs. post-swap; $z=1.27, p=0.21$ ).

Experiment 3: transfer of wax and resin odours to workers

Treatment of nestmate bees with either wax or resin lowered their acceptance rates significantly to that of non-nestmates, irrespective of whether the wax/resin originated from a nestmate or non-nestmate hive (Fig. $2 ; 84.1 \%$ vs. $33.3 \%$, $z=4.78, p=<0.001 ; 84.1 \%$ vs. $42.0 \%, z=4.71, p=<0.001$; $84.1 \%$ vs. $25.7 \%, z=5.27, p=<0.001 ; 84.1 \%$ vs. $18.6 \%$, $z=6.81, p=<0.001)$. Acceptance of non-nestmates remained low regardless of treatment $(16.7 \%$ vs. $14.3 \%, z=0.41$, $p=0.67 ; 16.7 \%$ vs. $18.6 \%, z=-0.33, p=0.73 ; 16.7 \%$ vs. $18.2 \%, z=-0.26, p=0.79 ; 16.7 \%$ vs. $5.7 \%, z=2.05$, $p=0.040$ ). Interestingly, there were no pronounced differences between resin and wax sourced from nestmate and nonnestmate hives. The acceptance rates of nestmates treated with nestmate or non-nestmate wax did not differ significantly (33.3\% vs. $42.0 \%, z=-0.86, p=0.39)$, reflecting what we found for non-nestmates with the same treatments (14.3\% vs. $18.6 \%, z=-0.68, p=0.49$ ). Similarly, the acceptance rates of nestmates treated with nestmate or non-nestmate resin did not differ significantly $(18.6 \%$ vs. $25.7 \%, z=0.84, p=0.39)$. However, non-nestmates treated with non-nestmate resin were rejected to a greater extent than nestmates treated with non-nestmate resin $(5.7 \%$ vs. $18.2 \%, z=2.14, p=0.03)$.

Experiment 4: one-way transfer of resin between hives

After the unidirectional transfer of resin, the acceptance rate of nestmates dropped by $37.5 \%$ for resin donor hives (from $81.9 \%$ to $44.4 \%, z=-3.51, p=<0.001$; Fig. 3 ), while only a decline of $4.5 \%$ was seen for resin recipient hives (from $82.3 \%$ to $77.8 \%, z=-0.36, p=0.71)$. Conversely, for nonnestmates, a non-significant increase of $1.6 \%$ in acceptance rates was seen in donor hives (from $14.3 \%$ to $15.9 \%$, $z=0.35, p=0.72$ ), while a significant rise of $21.6 \%$ was observed for recipient hives (from $6.2 \%$ to $27.8 \%, z=2.75$, $p=0.005$ ). This effect was independent of the resin source, that is, acceptance rates did not differ between non-nestmates from the paired hive and non-nestmates from other hives $(z=-1.03, p=0.30)$. The overall trends were somewhat inconsistent amongst the hives with notable variation apparent (see "Electronic supplementary material").

\section{Discussion}

The results of the first experiment involving transferral of bee-derived odours confirm the exceptional recognition abilities of T. angustula compared to other studied species, with combined recognition errors of $10 \%$. Acceptance rates of nestmates treated with non-nestmate odour were greatly and significantly reduced, while the small positive effect on acceptance from treating non-nestmates with nestmate odour was non-significant. Together with recent studies on the honeybee (Ratnieks et al. 2011), the stingless bee $F$. varia (Couvillon and Ratnieks 2008) and Camponotus ants (Guerrieri et al. 2009), these results support the idea that the odour space of a particular colony odour utilised in nestmate recognition is complex and multidimensional. In particular, a multidimensional odour space helps explain why it is far easier for a nestmate odour to diverge from the colony odour rather than a non-nestmate odour to converge on the colony odour of the guards (Ratnieks et al. 2011). In this framework, the average chemical distance that an individual is moved following odour transfer is enough to make a nestmate unacceptable but not enough to make a non-nestmate acceptable even though both changes are equal in chemical distance. Nestmate odours are chemically similar and therefore fall within the overall colony odour profile. Any deviation therefore is easily recognised; however, non-nestmate odours are sufficiently distant in chemical space that the same deviation, even towards the nestmate odour profile, is not enough to fall within the colony odour profile (for a visual representation, see Fig. 3 in Ratnieks et al. 2011).

Swapping of entrance tubes had no effect on acceptance and allows us to reject the hypothesis that accurate nestmate recognition in $T$. angustula is due to the wax entrance tube serving as a convenient or immediate template for colony odour. Our data show a small but non-significant 3\% increase in the acceptance rates of non-nestmates introduced after tube swapping, which is only $1 / 30$ th of the $90 \%$ difference seen between the acceptance of untreated nestmates (94.6\%) and untreated non-nestmates (4.5\%) observed in our first experiment. Our results also show no significant change in acceptance rates with time, from 1 to $24 \mathrm{~h}$ post-tube swap. We would expect to see an effect in this time frame as swapping of wax combs in honeybees leads to a change in behaviour within hours (Couvillon et al. 2007a).

While the first two experiments both provided very clear findings, the results of the subsequent two were less clear. Following treatment of worker bees with wax and resin odours, we found no difference between acceptance rates of bees treated with nestmate wax or resin versus nonnestmate wax or resin. If wax or resin serves as a source of colony odour, we would have expected to see a disparity between nestmates treated with their own wax or resin versus non-nestmate wax or resin, as seen in the first experiment where the odours in the vial were derived from live bees. Acceptance rates of nestmates dramatically 
Fig. 3 Experiment 4: Nestmate and non-nestmate workers were introduced to six discriminator hives, both before and after a unidirectional resin transfer. Three discriminator hives were resin donors and three were resin recipients, forming three pairs. Exact percentage acceptance rates and sample sizes are given above the bars. Statistically significant differences are indicated $(* * p<0.01$, $* * * p<0.001$ )

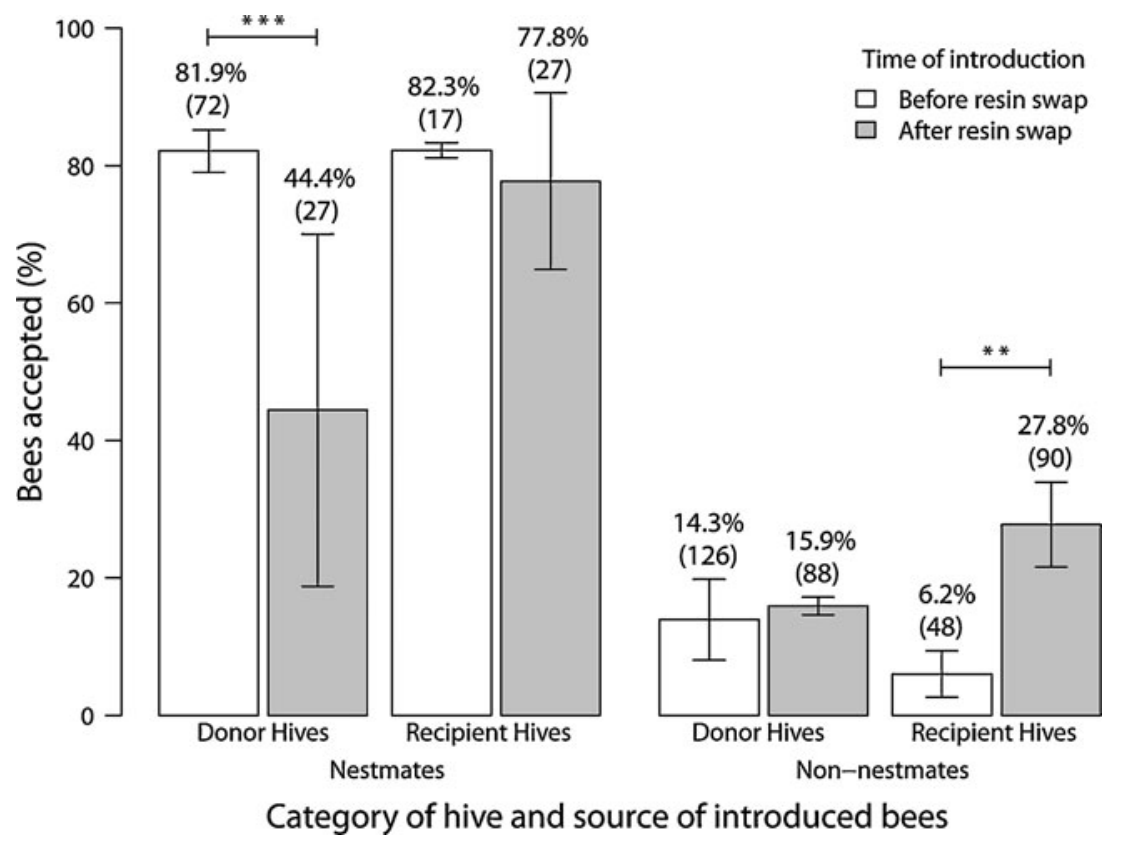

thing else may be occurring which our experiment was unable to reveal and therefore warrants further investigation.

The behaviour shown by the guards of resin donor hives is also puzzling. The post-transfer decline in acceptance of nestmates may be a response to the loss of the colony's entire resin store, but if this were the case we would predict a simultaneous increase in rejection rates of non-nestmates, which was not seen. The high variation in acceptance rates evident within both the donor and recipient colonies is perhaps indicative of guard confusion. Indeed this was conspicuous with guards exhibiting frequent and intense antennation with greater periods of time preceding rejection (S.M. Jones, personal observation). This lack of consistency in changes in acceptance rates among the discriminator colonies was also apparent in the wax and resin odour transfer experiment and is notably different from the consistent changes seen in the first two experiments. Our findings appear to show that $T$. angustula do not use pure resin as a source of cues for nestmate recognition. Several studies have failed to identify the presence of terpenoids on the wings of various Neotropical stingless bees (Abdalla et al. 2003; Jungnickel et al. 2004; Kerr et al. 2004; Nunes et al. 2008). To our knowledge, no study has yet analysed the cuticular chemical profiles of $T$. angustula but it would be surprising if terpenoids were absent when we know that resin, which is a rich source of terpenoids (Velikova et al. 2000; Sawaya et al. 2006), is found on the thorax of many foragers (J.S. van Zweden, unpublished data) and is universally present within the hive both in resin piles or mixed with wax as cerumen (S.M. Jones, personal observation; Michener 1974).

It is possible that the inconsistent results seen for the resin transfer experiment may have arisen because the resin transfer. This marked variation suggests that some- 
terpenoid composition of the pure resin we collected from the resin piles does not reflect the terpenoid profile present on the cuticles of the bees. Leonhardt et al. (2011) showed that terpenoids present on the cuticles of six different Paleotropical stingless bees differed from those found on nest material. Leonhardt et al. (2011) were also able to show that, from a total of 1,117 terpenoids available in stored resin, only $10 \%$ (105) were actually present on the cuticle of the Paleotropical stingless bee, Tetragonilla collina. To explain this, Leonhardt et al. (2011) proposed a hypothesis whereby stingless bees are able to perform some form of post-collection manipulation of resin terpenoids to ensure odour constancy. Resin stored by colonies of T. angustula from across Brazil was found to have a remarkably consistent composition, regardless of location (Sawaya et al. 2006). Therefore, for terpenoids to function as cues for nestmate recognition, quantitative differences between a discrete set of these compounds must be apparent among colonies and this would have to be achieved by some form of post-collection manipulation. Confirmation of whether terpenoids can be manipulated in this manner or indeed function as suitable recognition cues will require further behavioural and analytical study. A more parsimonious explanation may be that resin simply does not function as a primary source of recognition cues in T. angustula. An inherent problem with using collected materials, such as resin or food, as odour cues is the likelihood that the availability of the sources will change with time (Downs et al. 2000, 2001). Once a bee collects new material which is not consistent with its colony odour, there is a strong possibility that it will be rejected. For example, floral odours, most of which are terpenoids, were found to have no function in honey bee nestmate recognition (Downs et al. 2000).

Overall, our results confirm the accuracy of the nestmate recognition system in $T$. angustula. When results of the controls from the first three experiments were combined, a typical average of $10 \%$ was observed for both acceptance and rejection errors, giving a total error rate of $20 \%$. Despite the variation that exists between colonies and studies, the error rate remains considerably lower than those reported for honey bees (Downs and Ratnieks 1999, 2000; Couvillon et al. 2007a, 2008, 2009, 2010) and lower than all stingless bee species studied to date (Breed and Page 1991; Buchwald and Breed 2005; Couvillon and Ratnieks 2008). Although our results do not show how T. angustula achieves this accuracy, we have ruled out one strong contender: the wax entrance tubes of $T$. angustula nests appear to play no role in nestmate recognition. Our results from the resin odour treatment and resin transfer experiments suggest that odours acquired directly from resin also serve no function as nestmate recognition cues, although the observed shifts in the acceptance threshold seen for the resin transfer suggest a possible secondary role. However, the variation and inconsistency of our results in the last two experiments together highlight the need for future chemical analysis of resin stores, cerumen and the cuticular profiles of worker bees. Indeed the results of a recent study by Nunes et al. (2011) suggest that cerumen may be a source of recognition cues, used by colony members of the stingless bee $F$. varia. It also remains to be seen whether this proficient recognition system has evolved as a result of low genetic variability or high parasite pressure.

Acknowledgements We thank Dr. Paulo Noguiera-Neto for his hospitality at Fazenda Aretuzina and giving us permission to manipulate and study his bee colonies. We also thank Dr. Margaret Couvillon, Associate Editor, Dr William Hughes and anonymous referees for their comments and criticisms. S.M.J. was funded by a GTA grant from the University of Sussex. J.S.v.Z. was supported by a postdoctoral fellowship from the Danish Council for Independent Research (09066595) and C.G. by a postdoctoral fellowship from the Swiss National Science Foundation (SNSF grant PBBEP3-123648). T.C. was supported by a Ph.D. studentship from BBSRC. FAPESP provided funding for C.M. (07/50218-1), D.A.A. (05/58093-8; 10/19717-4), P.N.S (07/03864-5), V.L.I.F. (04/15801-0) and F.L.W.R (08/57782-2).

\section{References}

Abdalla FC, Jones GR, Morgan ED, da Cruz-Landim C (2003) Comparative study of the cuticular hydrocarbon composition of Melipona bicolor Lepeletier, 1836 (Hymenoptera, Meliponini) workers and queens. Genet Mol Res 2:191-199

Bates D (2007) lme4: Linear mixed-effects models using S4 classes. R package version $0.99875-7$

Beale G (1990) Self and nonself recognition in the ciliate protozoan Euplotes. Trends Genet 6:137-139

Bell WJ, Breed MD, Richards KW, Michener CD (1974) Social, stimulatory and motivational factors involved in intraspecific nest defense of a primitively eusocial halictine bee. J Comp Physiol 93:173-181

Bolker BM, Brooks ME, Clark CJ, Geange SW, Poulsen JR, Stevens MHH, White J-SS (2009) Generalized linear mixed models: a practical guide for ecology and evolution. Trends Ecol Evol 24:127-135

Breed MD (1983) Nestmate recognition in honey bees. Anim Behav 31:86-91

Breed MD, Page RE (1991) Intra- and interspecific nestmate recognition in Melipona workers (Hymenoptera: Apidae). J Insect Behav 4:463-469

Breed MD, Garry MF, Pearce AN, Hibbard BE, Bjostad LB, Page J (1995) The role of wax comb in honey bee nestmate recognition. Anim Behav 50:489-496

Breed MD, Diaz PH, Lucero KD (2004) Olfactory information processing in honeybee, Apis mellifera, nestmate recognition. Anim Behav 68:921-928

Buchwald R, Breed MD (2005) Nestmate recognition cues in a stingless bee, Trigona fulviventris. Anim Behav 70:1331-1337

Butler CG, Free JB (1952) The behaviour of worker honey bees at the hive entrance. Behaviour 4:262-292

Couvillon MJ, Ratnieks FLW (2008) Odour transfer in stingless bee marmelada (Frieseomelitta varia) demonstrates that entrance guards use an "undesirable-absent" recognition system. Behav Ecol Sociobiol 62:1099-1105 
Couvillon MJ, Caple JP, Endsor SL, Kärcher MH, Russell TF, Storey DE, Ratnieks FLW (2007a) Nest-mate recognition template of guard honeybees (Apis mellifera) is modified by wax comb transfer. Biol Lett 3:228-230

Couvillon MJ, Wenseleers T, Imperatriz-Fonseca VL, Nogueira-Neto P, Ratnieks FLW (2007b) Comparative study in stingless bees (Meliponini) demonstrates that nest entrance size predicts traffic and defensivity. J Evol Biol 21:194-201

Couvillon MJ, Robinson EJH, Atkinson B, Child L, Dent KR, Ratnieks FLW (2008) En garde: rapid shifts in honeybee, Apis mellifera, guarding behaviour are triggered by onslaught of conspecific intruders. Anim Behav 76:1653-1658

Couvillon MJ, Roy GGF, Ratnieks FLW (2009) Recognition errors by honey bee (Apis mellifera) guards demonstrate overlapping cues in conspecific recognition. J Apicult Res 48:225-232

Couvillon MJ, Barton SN, Cohen JA, Fabricius OK, Kärcher MH, Cooper LS, Silk MJ, Helanterä H, Ratnieks FLW (2010) Alarm pheromones do not mediate rapid shifts in honey bee guard acceptance threshold. J Chem Ecol 36:1306-1308

d'Ettorre P, Heinze J (2005) Individual recognition in ant queens. Curr Biol 15:2170-2174

d'Ettorre P, Wenseleers T, Dawson J, Hutchinson S, Boswell T, Ratnieks FLW (2006) Wax combs mediate nestmate recognition by guard honeybees. Anim Behav 71:773-779

Downs SG, Ratnieks FLW (1999) Recognition of conspecifics by honeybee guards uses nonheritable cues acquired in the adult stage. Anim Behav 58:643-648

Downs SG, Ratnieks FLW (2000) Adaptive shifts in honey bee (Apis mellifera L.) guarding behavior support predictions of the acceptance threshold model. Behav Ecol 11:326-333

Downs SG, Ratnieks FLW, Jeffries SL, Rigby HE (2000) The role of floral oils in the nestmate recognition system of honey bees (Apis mellifera L.). Apidologie 31:357-365

Downs SG, Ratnieks FLW, Badcock NS, Mynott A (2001) Honeybee guards do not use food-derived odours to recognize non-nest mates: a test of the odour convergence hypothesis. Behav Ecol 12:47-50

Espelie KE, Wenzel JW, Chang G (1990) Surface lipids of social wasp Polistes melricus say and its nest and nest pedicel and their relation to nestmate recognition. J Chem Ecol 16: 2229-2241

Gastauer M, Campos LAO, Wittmann D (2011) Handling sticky resin by stingless bees (Hymenoptera, Apidae). Rev Bras Entomol 55:234-240

Glass NL, Kaneko I (2003) Fatal attraction: non-self recognition and heterokaryon incompatibility in filamentous fungi. Eukaryot Cell 2:1-8

Grüter C, Kärcher MH, Ratnieks FLW (2011) The natural history of nest defence in a stingless bee, Tetragonisca angustula (Latreille) (Hymenoptera: Apidae), with two distinct types of entrance guards. Neotrop Entomol 40:55-61

Guerrieri FJ, Nehring V, Jørgensen CG, Nielsen J, Galizia CG, d'Ettorre P (2009) Ants recognize foes and not friends. P R Soc B 276:2461-2468

Janeway CA, Medzhitov R (2002) Innate immune recognition. Ann Rev Immunol 20:197-216

Jungnickel H, da Costa AJS, Tentschert J, Patricio EFLRA, ImperatrizFonseca VL, Drijfhout F, Morgan ED (2004) Chemical basis for inter-colonial aggression in the stingless bee Scaptotrigona bipunctata (Hymenoptera: Apidae). J Insect Physiol 50:761-766

Kärcher MH, Ratnieks FLW (2009) Standing and hovering guards of the stingless bee Tetragonisca angustula complement each other in entrance guarding and intruder recognition. J Apicult Res 48:209-214

Kerr WE, Jungnickel H, Morgan ED (2004) Workers of the stingless bee Melipona scutellaris are more similar to males than to queens in their cuticular compounds. Apidologie 35:611-618
Lacy RC, Sherman PW (1983) Kin recognition by phenotype matching. Am Nat 121:489-512

Leonhardt SD, Blüthgen N, Schmitt T (2009) Smelling like resin: terpenoids account for species-specific cuticular profiles in Southeast-Asian stingless bees. Insectes Soc 56:157-170

Leonhardt SD, Schmitt T, Blüthgen N (2011) Tree Resin Composition, Collection Behavior and Selective Filters Shape Chemical Profiles of Tropical Bees (Apidae: Meliponini). PLoS ONE 6:e23445

Leonhardt SD, Blüthgen N, Schmitt T (2011) Chemical profiles of body surfaces and nests from six Bornean stingless bee species. J Chem Ecol 37:98-104

Martin SJ, Helanterä H, Drijfhout FP (2008) Colony-specific hydrocarbons identify nest mates in two species of Formica ant. J Chem Ecol 34:1072-1080

Michener CD (1974) The social behaviour of the bees. Harvard University Press, Cambridge

Nasrallah JB (2002) Recognition and rejection of self in plant reproduction. Science 296:305-308

Nogueira-Neto P (1997) Vida e criação de abelhas indigenas sem ferrão. Editora Nogueirapis, São Paulo

Nunes TM, Nascimento FS, Turatti IC, Lopes NP, Zucchi R (2008) Nestmate recognition in a stingless bee: does the similarity of chemical cues determine guard acceptance? Anim Behav $75: 1165-1171$

Nunes TM, Mateus S, Turatti IC, Morgan ED, Zucchi R (2011) Nestmate recognition in the stingless bee Frieseomelitta varia (Hymenoptera, Apidae, Meliponini): sources of chemical signals. Anim Behav 81:463-467

Ozaki M, Wada-Katsumata A, Fujikawa K, Iwasaki M, Yokohari F, Satoji Y, Nisimura T, Yamaoka R (2005) Ant nestmate and non-nestmate discrimination by a chemosensory sensillum. Science 309:311-314

Ratnieks FLW (1991) Facultative sex allocation biasing by workers in social Hymenoptera. Evolution 45:281-292

Ratnieks FLW, Kärcher MH, Firth V, Parks D, Richards A, Richards P, Helanterä H (2011) Acceptance by honey bee guards of nonnestmates is not increased by treatment with nestmate odours. Ethology 117:655-663

R Development Core Team (2009) R: A language and environment for statistical computing. R Foundation for Statistical Computing, Vienna, Austria

Reeve HK (1989) The evolution of conspecific acceptance thresholds. Am Nat 133:407-435

Roubik DW (2006) Stingless bee nesting biology. Apidologie 37: 124-143

Sawaya ACHF, Cunha IBS, Marcucci MC, Rodrigues RFD, Eberlin MN (2006) Brazilian propolis of Tetragonisca angustula and Apis mellifera. Apidologie 37:398-407

Sherman PW, Reeve HK, Pfennig DW (1997) Recognition systems: behavioural ecology: an evolutionary approach, 4th edn. WileyBlackwell, Cambridge, pp 69-96

Tibbetts EA (2002) Visual signals of individual identity in the wasp Polistes fuscatus. P Roy Soc Lond B Bio 269:1423-1428

van Veen JW, Sommeijer MJ (2000) Observations on gynes and drones around nuptial flights in the stingless bees Tetragonisca angustula and Melipona beecheii (Hymenoptera, Apidae, Meliponinae). Apidologie 31:47-54

van Zweden JS, d'Ettorre P (2010) Nestmate recognition in social insects and the role of hydrocarbons: insect hydrocarbons. Cambridge University Press, Cambridge, pp 222-243

van Zweden JS, Grüter C, Jones SM, Ratnieks FLW (2011) Hovering guards of the stingless bee Tetragonisca angustula increase colony defensive perimeter as shown by intra- and inter-specific comparisons. Behav Ecol Sociobiol 65:1277-1282

Vander Meer RK, Saliwanchik D, Lavine B (1989) Temporal changes in colony cuticular hydrocarbon patterns of Solenopsis invictaimplications for nestmate recognition. J Chem Ecol 15:2115-2125 
Vander Meer RK, Breed MD, Espelie KE, Winston M (1998) Nestmate recognition in ants. In: Vander Meer RK (ed) Pheromone communication in social insects: ants, wasps, bees and termites. Westview, Oxford

Velikova M, Bankova V, Marcucci MC, Tsvetkova I, Kujumgiev A (2000) Chemical composition and biological activity of propolis from Brazilian Meliponinae. Z Naturforsch C 55:785-789

Wille A (1983) Biology of the stingless bees. Ann Rev Entomol 28:41-64
Wittmann D (1985) Aerial defense of the nest by workers of the stingless bee Trigona (Tetragonisca) angustula (Latreille) (Hymenoptera: Apidae). Behav Ecol Sociobiol 16:111-114

Wittmann D, Radtke R, Zeil J, Lübke G, Francke W (1990) Robber bees (Lestrimelitta limao) and their host chemical and visual cues in nest defense by Trigona (Tetragonisca) angustula (Apidae: Meliponinae). J Chem Ecol 16:631-641

Zuur AF, Ieno EN, Walker NJ, Saveliev AA, Smith GM (2009) Mixed effects models and extensions in ecology with R. Springer, New York, p 574 